\title{
Prevalência de desnutrição em crianças residentes no Município de Embu, São Paulo, Brasil, 1996-1997
}

\author{
Prevalence of malnutrition among children \\ in Embu, São Paulo State, Brazil, 1996-1997
}

\begin{abstract}
Maria Wany Louzada Strufaldi 1
Rosana Fiorini Puccini 1

Glaura César Pedroso 1

Edina Mariko Koga da Silva 1

Nilza Nunes da Silva 2
\end{abstract}

\footnotetext{
1 Departamento de Pediatria, Universidade Federal de São Paulo. Rua Botucatu 598, São Paulo, SP 04023-090, Brasil. edina.dmed@epm.br 2 Departamento de Epidemiologia, Faculdade de Saúde Pública, Universidade de São Paulo. Av. Dr. Arnaldo 715, São Paulo, SP 01246-904, Brasil. nndsilva@usp.br
}

\begin{abstract}
This paper focused on the nutritional profile of children in the municipality of Embu, São Paulo State, Brazil, in 1996-1997, to identify vulnerable population segments that require specific action by health services. The sample consisted of 320 children $\leq 5$ years of age distributed into four socioeconomic strata. The indices were expressed as z-scores: weight/age (W/A), height/age (H/A), and weight/height (W/H) to analyze the nutritional status, and the reference for normality was the NCHS curve. In all population strata and age groups, the height/age index was the most frequently affected, while the weight/height index had the fewest deficits. No statistically significant differences were observed in children's nutritional status between the four population strata or between the different age groups. Children with low birth weight showed the highest prevalence of deficits in all indices. In the municipality, the frequency of deficits were: $H / A<2 z: 7.1 \%, W / H<2 z: 0.2 \%$, and $W / A<2 z: 2.9 \%$. Height deficit can be used as an early warning, considering that loss observed over the course of years has future consequences.
\end{abstract}

Key words Nutrition Disorders; Anthropometry; Nutrition Surveys

Resumo O presente trabalho teve como objetivo descrever o perfil nutricional de crianças residentes no Município de Embu, São Paulo, Brasil, em 1996-1997, visando a identificar segmentos populacionais mais vulneráveis que demandassem atuação específica dos serviços de saúde. A amostra consistiu em 320 crianças de zero a cinco anos, distribuídas em quatro estratos de condições de vida. Considerou-se os índices expressos em z escores: P/I, E/I e P/E, para avaliação antropométrica e a curva NCHS como referência. Em todos os estratos e em todas as faixas de idade, o índice E/I foi o mais freqüentemente comprometido e o P/E apresentou menores freqüências de déficits. Não se observaram diferenças estatisticamente significantes entre os quatro estratos e entre as faixas de idade. As crianças nascidas com baixo peso apresentaram maiores prevalências de déficits, considerando-se todos os índices. As freqüencias de déficits para o município foram: $P / I<2 z: 2,9 \%, E / I<2 z: 7,1 \%$ e $P / E<2 z: 0,2 \%$. O déficit estatural pode servir como alerta, considerando que perdas ao longo dos anos têm reflexos no futuro.

Palavras-chave Transtornos Nutricionais; Antropometria; Inquéritos Nutricionais 


\section{Introdução}

O diagnóstico e a vigilância das condições nutricionais das crianças constituem forma prática e sensível de avaliar, simultaneamente, condições e tendências da saúde infantil, o grau com que vêm sendo atendidos os direitos humanos elementares da população e as possibilidades de desenvolvimento da sociedade (Buvinich, 1998). Os distúrbios do estado de saúde e nutrição durante a infância podem ser ocasionados por múltiplas condições, predominando, sobretudo nos países em desenvolvimento, deficiências alimentares e infecções de repetição, estas largamente dependentes das condições gerais de vida e do acesso a necessidades básicas como alimentos, moradia e assistência à saúde (Monteiro et al., 2000a; Monteiro \& Conde, 2000).

O comprometimento nutricional está associado à maior incidência e gravidade de doenças infecciosas, elevação nas taxas de mortalidade na infância, retardo no desenvolvimento psicomotor, dificuldades escolares, diminuição da estatura e da capacidade reprodutiva na idade adulta (Monteiro \& Conde, 2000). Segundo relatório publicado pela Organização Mundial da Saúde (OMS), em 2000, nos países em desenvolvimento, $35 \%$ das crianças abaixo dos cinco anos de idade apresentavam déficit de estatura (estatura para idade menor que dois desvios-padrão), sendo que na América do Sul a prevalência deste déficit era de 9,3\% (Onis et al., 2000).

A antropometria tem sido amplamente utilizada, com sucesso, na avaliação da saúde e de riscos nutricionais, especialmente em crianças, auxiliando na definição de prioridades no planejamento, implementação e avaliação de programas (WHO, 1995). Três índices antropométricos são habitualmente usados: P/I (peso para idade), E/I (estatura para idade) e P/E (peso para estatura). Embora estes índices, isoladamente, não estabeleçam o processo específico que levou uma ou um grupo de crianças à desnutrição, eles são freqüentemente utilizados para representar os graus de carências aos quais as crianças estão submetidas.

O presente estudo teve como objetivo principal conhecer os índices antropométricos da população de crianças de zero a cinco anos de idade, residentes no Município de Embu, em 1996. O perfil nutricional e as condições de saúde destas crianças são informações valiosas para o planejamento de ações, visando a prevenção e a programação de cuidados dirigidos às crianças com déficits já instalados.

\section{Metodologia}

Este estudo é parte integrante do projeto Morbidade Infantil e Utilização de Serviços de Saú$d e$, financiado pela Fundação de Amparo à Pesquisa do Estado de São Paulo (FAPESP - Projeto 95/3438-7) e realizado por profissionais da Universidade Federal de São Paulo (UNIFESP), da Faculdade de Saúde Pública da Universidade de São Paulo e da Secretaria Municipal de Saúde do Embu (Silva et al., 1997).

O Município de Embu, situado na Região Metropolitana de São Paulo, dista $27 \mathrm{~km}$ do centro da capital do estado, tem uma área de 76 km² e sua população, em 2000, era de 194.879 habitantes. Em 1996, seu coeficiente de mortalidade infantil era de 28,12 por mil nascidos vivos e, em 2000 , declinou para 16,96 por mil nascidos vivos, sendo que dois terços destes óbitos correspondem à mortalidade neonatal (Fundação Seade, 2001a). A Rede Municipal de Saúde, em 1996, estava constituída por nove Unidades Básicas de Saúde (UBS) e dois Pronto Socorros (PS). Não havia leitos hospitalares no município. A Escola Paulista de Medicina (EPM/UNIFESP) atua no Embu desde 1970, envolve vários de seus departamentos e disciplinas e mantém uma participação ativa no Conselho Municipal de Saúde desde 1991 (UNIFESP, 2001).

\section{Processo de amostragem e coleta de dados}

Devido a especificidades da morbi-mortalidade e padrão de utilização dos serviços de saúde, foram consideradas duas populações independentes: crianças menores de um ano e de um a quatro anos residentes no Município de Embu em 1996. Dispondo de arquivos de dados do censo demográfico de 1991 (IBGE, 1991), a população foi estratificada em agrupamentos homogêneos definidos segundo condições de moradia e situação sócio-econômica da família. Os 135 setores censitários que compõem a área geográfica do município foram reunidos através da técnica de análise de agrupamentos (Frei, 1997). As variáveis: tipo de domicílio (casa isolada, apartamento, aglomerado subnormal); abastecimento de água (presença de canalização interna ligada à rede geral); coleta de lixo; instalação sanitária dentro do domicílio; chefes de família analfabetos; chefes de família com 15 anos e mais de instrução; rendimento mensal do chefe inferior a meio salário mínimo; domicílios com até quatro moradores; domicílios com dez e mais moradores, foram selecionadas para estratificação dos setores que resultaram em quatro estratos com os seguintes perfis: 
(a) Estrato 1: com 17 setores censitários, envolvia a população residente em pequenas chácaras e condomínios, incluindo caseiros e suas famílias; este estrato apresentava melhores condições de ambiente, renda, moradia e escolaridade. A maioria de seus setores estava concentrada na região turística do município.

(b) Estratos 2 e 3: com 67 e 34 setores censitários, respectivamente, refletiam as condições ambientais predominantes no município; embora semelhantes entre si, foram mantidos separados devido à existência de favelas no estrato 3 .

(c) Estrato 4: com 17 setores censitários, todas as moradias foram classificadas como aglomerados subnormais (favelas). A escolaridade e as condições ambientais, de renda e de moradia eram as piores do município.

O processo de sorteio adotado foi o de conglomerados em dois estágios. No primeiro, dez setores censitários foram sorteados em cada estrato, sob o critério de partilha proporcional aos seus tamanhos (Kish, 1965). No segundo estágio, foram sorteados domicílios dentro de cada setor anteriormente selecionado. O questionário foi respondido pela mãe ou por seu substituto, mediante entrevista realizada no domicílio. A amostra nesta primeira etapa foi constituída de 483 crianças menores de um ano e 616 crianças de um a quatro anos de idade, e a coleta destes dados foi realizada no período de agosto a novembro de 1996.

Na segunda etapa do trabalho, uma subamostra de cem crianças para cada estrato (total de 400 crianças) foi sorteada para avaliação nutricional, dosagem de hemoglobina e exame físico. O trabalho de campo desta segunda etapa, foi realizado no período de novembro de 1996 a julho de 1997 e, por este motivo, parte das crianças de quatro anos já havia completado cinco. Para a análise dos dados desta segunda etapa, a amostra foi considerada em seu conjunto, isto é, foram agrupadas as crianças menores de um ano e crianças de 1 a 5 anos. Os procedimentos desta segunda etapa foram realizados após assinatura de consentimento informado pelos responsáveis, sendo que, mesmo sem recusa, em 80 (20,0\%) crianças não foram realizados os exames devido à mudança de domicílio. Assim, a amostra final, para este estudo foi composta de 320 crianças. As Tabelas 1 e 2 descrevem, respectivamente, as características sociodemográficas e condições de assistência à saúde das famílias destas crianças. Não há diferenças, estatisticamente significantes, entre as freqüências dos indicadores referentes à utilização de serviços de saúde, ainda que se observe uma tendência de menor acesso no estrato 4 quanto à realização do pré-natal e a planos privados ou convênios. O pré-natal foi definido como adequado, considerando-se os seguintes critérios: seis ou mais consultas, início no primeiro trimestre de gestação, realização de exames laboratoriais, aferição de pressão arterial em uma ou mais consultas, realização de exame das mamas em uma ou mais consultas e realização de pelo menos um exame de ultrassonografia. Com exceção do peso ao nascer, não foi realizada análise para investigação de associação entre as variáveis destas tabelas e os déficits nutricionais.

\section{Variáveis do estudo}

\section{- Estado nutricional}

a) Peso: para crianças menores de dois anos foi utilizada balança com cesto, digital, com capacidade de até 15 quilos e, para maiores de dois anos, utilizou-se uma balança do tipo plataforma, digital, com capacidade de até 150 quilos.

b) Estatura: para crianças menores de dois anos, foi utilizado antropômetro de madeira do tipo horizontal, com régua graduada em milímetros; a criança era medida em decúbito dorsal. Para os maiores de dois anos a medida foi tomada utilizando-se fita métrica, milimetrada, presa à parede e com esquadro de madeira, com trava.

Foram utilizados três índices antropométricos: peso para idade $(\mathrm{P} / \mathrm{I})$, estatura para idade $(\mathrm{E} / \mathrm{I})$ e peso para estatura $(\mathrm{P} / \mathrm{E})$. A curva de referência foi a do National Center for Health Statistics (NCHS, 1977), e a avaliação nutricional utilizou a distribuição dos índices expressos em escores $z$, considerado-se que crianças com índices inferiores a dois desvios padrão apresentavam déficits antropométricos (WHO, 1986, 1995).

\section{- Peso, idade}

Obtidos por informação da mãe na entrevista.

\section{- Estratos de condição de vida}

Já descritos.

\section{- Análise estatística}

Para processamento, entrada e análise dos dados, utilizou-se o programa Epi Info - versão 6.04, incluindo o módulo CSAMPLE, que permite ponderação dos dados, e o EPINUT, para avaliação nutricional. A amostragem por conglomerados em dois estágios introduziu dife- 
Características sócio-demográficas das famílias de crianças de zero a cinco anos. Embu, São Paulo, Brasil, 1996.

\begin{tabular}{|c|c|c|c|c|c|c|c|c|c|c|c|c|}
\hline \multirow{2}{*}{ Características } & \multicolumn{2}{|c|}{ Estrato 1} & \multicolumn{2}{|c|}{ Estrato 2} & \multicolumn{2}{|c|}{ Estrato 3} & \multicolumn{2}{|c|}{ Estrato 4} & \multicolumn{4}{|c|}{ Total } \\
\hline & $\%$ & EP & $\%$ & EP & $\%$ & EP & $\%$ & $\mathrm{EP}$ & $\mathrm{n}$ & $\%$ & EP & IC $95 \%$ \\
\hline Renda familiar per capita $<1 \mathrm{SM}$ & 45,2 & 8,9 & 62,8 & 6,6 & 44,0 & 8,5 & 64,2 & 5,5 & 172 & 59,0 & 5,2 & $48,8-69,2$ \\
\hline Idade da mãe $<20$ anos & 13,6 & 7,4 & 5,6 & 2,3 & 3,6 & 1,9 & 12,3 & 2,5 & 36 & 6,4 & 1,8 & $1,9-9,9$ \\
\hline Mães alfabetizadas & 86,6 & 7,8 & 93,7 & 2,9 & 90,9 & 3,5 & 83,2 & 4,5 & 284 & 91,9 & 2,3 & $87,3-96,5$ \\
\hline Mães que cursaram a 4 a série ou + & 59,7 & 11,0 & 50,2 & 4,1 & 55,3 & 3,5 & 50,3 & 5,9 & 173 & 51,6 & 2,9 & $45,9-57,3$ \\
\hline Chefes de família alfabetizados & 92,1 & 5,7 & 79,3 & 5,9 & 96,9 & 2,0 & 87,7 & 3,2 & 261 & 83,5 & 4,7 & $74,3-92,7$ \\
\hline $\begin{array}{l}\text { Chefes de família que cursaram } \\
\text { a } 4^{\text {a }} \text { série ou }+\end{array}$ & 59,2 & 10,4 & 34,4 & 7,5 & 65,4 & 7,1 & 57,5 & 9,0 & 145 & 44,4 & 5,9 & $32,7-56,0$ \\
\hline Mãe natural de Embu & 14,1 & 4,7 & 3,9 & 2,4 & 4,0 & 2,4 & 0,0 & 0,0 & 22 & 4,3 & 1,7 & $0,9-7,7$ \\
\hline Chefe de família natural de Embu & 17,5 & 4,9 & 0,0 & 0,0 & 5,6 & 3,6 & 0,0 & 0,0 & 19 & 1,9 & 0,7 & $0,6-3,2$ \\
\hline $\begin{array}{l}\text { Mães que residem em Embu } \\
\text { há } 5 \text { anos ou }+\end{array}$ & 72,9 & 9,1 & 61,5 & 8,1 & 64,9 & 10,4 & 65,9 & 4,1 & 221 & 63,1 & 5,8 & $51,8-74,4$ \\
\hline $\begin{array}{l}\text { Chefes de família que residem } \\
\text { em Embu há } 5 \text { anos ou }+\end{array}$ & 73,2 & 6,3 & 65,0 & 4,4 & 65,3 & 11,7 & 69,3 & 4,4 & 204 & 65,9 & 3,6 & $58,8-73,0$ \\
\hline Óbito de irmão $<5$ anos & 5,4 & 2,4 & 6,0 & 3,4 & 5,6 & 2,6 & 17,8 & 4,0 & 32 & 7,1 & 2,5 & $2,1-12,1$ \\
\hline Mãe que trabalha fora do lar & 18,1 & 4,5 & 32,8 & 8,2 & 18,9 & 6,2 & 21,9 & 5,8 & 69 & 28,6 & 6,4 & $16,2-41,1$ \\
\hline Mãe que trabalha no lar & 11,3 & 6,2 & 3,5 & 2,7 & 7,5 & 2,7 & 3,0 & 2,5 & 22 & 4,5 & 2,0 & $0,6-8,5$ \\
\hline Chefe de família que trabalha & 88,5 & 3,3 & 72,2 & 7,9 & 91,0 & 5,5 & 86,8 & 3,0 & 248 & 77,4 & 6,3 & $65,0-89,8$ \\
\hline \multicolumn{13}{|l|}{ Cuidados para com a criança } \\
\hline Mãe & 81,1 & 4,9 & 72,2 & 9,9 & 80,2 & 7,1 & 86,7 & 3,5 & 263 & 75,4 & 7,3 & $61,0-89,8$ \\
\hline Parente/vizinho & 12,1 & 5,0 & 19,0 & 5,9 & 12,4 & 4,2 & 10,4 & 3,1 & 39 & 16,8 & 4,4 & $8,0-25,5$ \\
\hline Creche & 6,8 & 1,5 & 8,8 & 4,9 & 7,4 & 3,2 & 2,9 & 1,7 & 18 & 7,9 & 3,5 & $0,9-14,8$ \\
\hline
\end{tabular}

EP: Erro padrão estimado; SM: Salário mínimo.

renças de probabilidade (f), independente de qualquer família ou criança da população pertencer à amostra sorteada. Para cada estrato o peso $\mathrm{w}$ foi calculado pelo inverso da probabilidade f. Ou seja, $w=1 /$ f. Na segunda etapa do trabalho, cem crianças foram sorteadas em cada estrato e um novo peso w' foi calculado a partir da fórmula $w^{\prime}=1$ /f'. A expressão matemática adotada para calcular f e f' decorreu do plano de amostragem adotado (Silva et al., 1997). A significância estatística na comparação entre os estratos, entre a faixa de peso ao nascer e idade, foi feita considerando as estimativas, erros padrão e intervalos de confiança maiores ou iguais a $95 \%$. Consideraram-se as diferenças como estatisticamente significantes, nos casos onde não havia sobreposição dos intervalos de confiança.

\section{Resultados}

Na Tabela 3, para cada estrato populacional são apresentadas as freqüências de déficits definidos como P/I, E/I ou P/E menores que $2 \mathrm{z}$. Em todos os estratos, o índice E/I foi o que apre- sentou maiores freqüências de déficits, porém, as diferenças observadas não foram estatisticamente significantes. Para os três índices, as freqüências de déficits foram maiores (sem significância estatística, pois os intervalos de confiança se sobrepunham) no estrato 4 em relação aos demais estratos e em relação ao município como um todo. Em todos os estratos populacionais, o índice $\mathrm{P} / \mathrm{E}$ foi o que apresentou menor freqüência de alteração, variando de zero (estratos 2 e 3 ) a $2,2 \%$ (estrato 4 ).

A Tabela 4 descreve a freqüência de déficits, segundo os mesmos índices antropométricos, para o município como um todo. Para todas as faixas de idade, o índice E/I apresentou maior freqüência de déficits (máximo de 14,3\% aos quatro anos de idade). Observa-se, mais uma vez, que o índice $\mathrm{P} / \mathrm{E}$ aproxima-se de zero, em qualquer faixa etária. Não foi observada distribuição crescente ou decrescente nos valores de cada índice, quando analisados em separado para cada faixa etária.

Na Tabela 5, encontra-se a distribuição de crianças com déficits (P/I, E/I e/ou P/E abaixo de $-2 z$ ), segundo o peso ao nascer. Verificamos que no grupo de crianças nascidas com baixo 
Assistência à saúde (famílias e crianças de zero a cinco anos). Embu, São Paulo, Brasil, 1996.

\begin{tabular}{|c|c|c|c|c|c|c|c|c|c|c|c|c|c|}
\hline \multirow[t]{2}{*}{ Características } & \multicolumn{2}{|c|}{ Estrato 1} & \multicolumn{2}{|c|}{ Estrato 2} & \multicolumn{2}{|c|}{ Estrato 3} & \multicolumn{2}{|c|}{ Estrato 4} & \multicolumn{5}{|c|}{ Total } \\
\hline & $\%$ & $\mathrm{EP}$ & $\%$ & $\mathrm{EP}$ & $\%$ & $\mathrm{EP}$ & $\%$ & EP & $n$ & $\%$ & EP & IC (95\%) & $\mathrm{n}^{*}$ \\
\hline Pré-natal & & & & & & & & & & & & & 320 \\
\hline Não fez & 3,4 & 1,9 & 9,9 & 7,7 & 4,9 & 2,6 & 11,2 & 5,0 & 17 & 6,7 & 3,7 & $-0,5-14,0$ & \\
\hline Fez adequado & 36,3 & 7,3 & 36,0 & 6,1 & 38,4 & 6,0 & 34,1 & 7,8 & 121 & 39,6 & 4,6 & $30,6-48,6$ & \\
\hline Fez inadequado & 60,3 & 7,3 & 54,1 & 12,9 & 56,7 & 6,9 & 54,7 & 6,0 & 182 & 53,7 & 8,0 & $38,0-69,4$ & \\
\hline Parto hospitalar & 90,2 & 7,3 & 99,6 & 0,4 & 96,8 & 2,7 & 94,0 & 3,5 & 308 & 98,1 & 0,9 & $96,3-99,8$ & 319 \\
\hline Peso ao nascer & & & & & & & & & & & & & 307 \\
\hline$<2.500 \mathrm{~g}$ & 18,1 & 5,7 & 6,7 & 3,2 & 12,0 & 4,6 & 10,8 & 3,6 & 43 & 8,6 & 2,6 & $3,4-13,7$ & \\
\hline 2.500 a $2.999 \mathrm{~g}$ & 25,5 & 8,2 & 28,4 & 5,8 & 16,5 & 5,5 & 25,0 & 4,1 & 70 & 26,1 & 4,4 & $17,4-34,7$ & \\
\hline$>3.000 \mathrm{~g}$ & 56,4 & 6,9 & 64,9 & 4,8 & 71,5 & 5,6 & 64,1 & 3,1 & 194 & 65,4 & 3,6 & $58,4-72,3$ & \\
\hline Matrícula (UBS) & 80,1 & 5,4 & 86,4 & 4,5 & 72,7 & 12,1 & 86,9 & 3,2 & 266 & 83,9 & 3,7 & $76,7-91,1$ & 320 \\
\hline $\begin{array}{l}\text { Acompanhamento de } \\
\text { rotina } \leq 15 \text { meses }\end{array}$ & 98,4 & 1,8 & 78,9 & 6,1 & 65,6 & 10,7 & 82,9 & 7,1 & 84 & 78,6 & 4,7 & $69,3-87,9$ & $103^{\star \star}$ \\
\hline Local de acompanhamento & & & & & & & & & & & & & $84^{\star \star \star}$ \\
\hline Rede Básica do Embu & 82,7 & 15,1 & 63,4 & 17,6 & 89,2 & 8,5 & 100,0 & 0,0 & 71 & 71,0 & 13,3 & $44,9-97,2$ & \\
\hline Público (Embu/outros) & 94,6 & 4,1 & 93,9 & 4,2 & 96,3 & 4,1 & 100,0 & 0,0 & 77 & 94,7 & 3,0 & $88,9-100,6$ & \\
\hline Privado/Convênio & 5,4 & 4,1 & 6,1 & 4,2 & 3,7 & 4,1 & 0,0 & 0,0 & 7 & 5,3 & 3,0 & $-0,6-11,1$ & \\
\hline Acesso a plano privado & 32,7 & 5,3 & 32,9 & 5,6 & 39,8 & 9,5 & 16,0 & 4,2 & 91 & 32,4 & 4,2 & $24,2-40,5$ & 300 \\
\hline
\end{tabular}

EP: Erro padrão estimado; UBS: Unidade Básica de Saúde.

* $\mathrm{n}$ = número total de respostas obtidas para cada variável, por ocasião da entrevista.

** número de crianças com idade $<15$ meses.

*** número de crianças $\leq 15$ meses que são acompanhadas na rotina de serviços de saúde.

Estado nutricional de crianças de zero a cinco anos, segundo estratos de condições de vida.

Embu, São Paulo, Brasil, 1996.

\begin{tabular}{|c|c|c|c|c|c|c|c|c|c|c|c|}
\hline \multirow{2}{*}{$\begin{array}{l}\text { Tipo de classificação } \\
(n=320)\end{array}$} & \multicolumn{2}{|c|}{ Estrato 1} & \multicolumn{2}{|c|}{ Estrato 2} & \multicolumn{2}{|c|}{ Estrato 3} & \multicolumn{2}{|c|}{ Estrato 4} & \multicolumn{3}{|c|}{ Total } \\
\hline & $\%$ & EP (IC 95\%) & $\%$ & EP (IC 95\%) & $\%$ & EP (IC 95\%) & $\%$ & EP (IC 95\%) & $n$ & $\%$ & EP (IC 95\%) \\
\hline \multicolumn{12}{|l|}{ z-score } \\
\hline $\mathrm{P} / \mathrm{I}<2 \mathrm{z}$ & 3,9 & $2,0(-0,1-7,9)$ & 2,0 & $1,4(-0,6-4,7)$ & 3,7 & $2,3(-0,8-8,1)$ & 8,5 & $3,3(2,0-14,9)$ & 18 & 2,9 & $1,1(-0,7-5,1)$ \\
\hline$E / I<2 z$ & 11,2 & $8,1(-4,7-27,1)$ & 5,7 & $3,3(-0,8-12,2)$ & 9,4 & $4,0(1,6-17,2)$ & 11,2 & $3,6(4,2-18,1)$ & 27 & 7,1 & $2,7(1,9-12,3)$ \\
\hline $\mathrm{P} / \mathrm{E}<2 \mathrm{z}$ & 0,6 & $0,6(-0,6-1,8)$ & 0,0 & 0,0 & 0,0 & 0,0 & 2,2 & $2,3(-2,4-6,7)$ & 5 & 0,2 & $0,2(-0,2-0,6)$ \\
\hline
\end{tabular}

EP: Erro padrão estimado. 
Tabela 4

Estado nutricional (z-score) de crianças de zero a cinco anos, segundo a idade.

Embu, São Paulo, Brasil, 1996.

\begin{tabular}{|c|c|c|c|c|c|c|}
\hline \multirow[t]{2}{*}{ Idade (anos) } & \multicolumn{2}{|c|}{$\mathrm{P} / \mathrm{I}<2 \mathrm{Z}$} & \multicolumn{2}{|c|}{$E / I<2 Z$} & \multicolumn{2}{|c|}{$P / E<2 Z$} \\
\hline & $\%$ & EP (IC 95\%) & $\%$ & EP (IC 95\%) & $\%$ & EP (IC 95\%) \\
\hline$<1(n=60)$ & 0,7 & $0,5(-0,4-1,7)$ & 9,9 & $6,3(-2,4-22,2)$ & 0,2 & $0,2(-0,2-0,6)$ \\
\hline $1(n=93)$ & 3,6 & $2,9(-2,1-9,4)$ & 5,6 & $3,4(-1,0-12,2)$ & 0,2 & $0,2(-0,1-0,5)$ \\
\hline $2(n=45)$ & 0,7 & $0,7(-0,7-2,1)$ & 7,1 & $5,1(-2,9-17,2)$ & 0,5 & $0,5(-0,5-1,4)$ \\
\hline $3(n=55)$ & 0,7 & $0,6(-0,4-1,8)$ & 2,6 & $2,0(-1,3-6,5)$ & 0,4 & $0,4(-0,4-1,1)$ \\
\hline $4(n=37)$ & 3,6 & $2,1(-0,5-7,7)$ & 14,3 & $10,8(-6,9-35,5)$ & 0,0 & 0,0 \\
\hline $5(n=30)$ & 8,4 & $5,4(-2,2-19,0)$ & 8,4 & $5,4(-2,2-19,0)$ & 0,0 & 0,0 \\
\hline Total & 2,9 & $1,1(0,7-5,1)$ & 7,1 & $2,6(2,0-12,3)$ & 0,2 & $0,2(-0,2-0,6)$ \\
\hline
\end{tabular}

EP: Erro padrão estimado.

Tabela 5

Estado nutricional (z-score) de crianças de zero a cinco anos, segundo o peso ao nascer.

Embu, São Paulo, Brasil, 1996.

\begin{tabular}{|c|c|c|c|c|c|c|c|c|c|c|c|c|}
\hline \multirow[t]{2}{*}{ Peso ao nascer } & \multicolumn{3}{|c|}{$P / I<2 Z$} & \multicolumn{3}{|c|}{$E / I<2 Z$} & \multicolumn{3}{|c|}{$\mathrm{P} / \mathrm{E}<2 \mathrm{Z}$} & \multicolumn{3}{|c|}{ (P/I ou $E / I$ ou $P / E<2 Z$ ) } \\
\hline & $\mathrm{n}$ & $\%$ & EP (IC 95\%) & $\mathrm{n}$ & $\%$ & EP (IC 95\%) & $\mathrm{n}$ & $\%$ & EP (IC 95\%) & $n$ & $\%$ & EP (IC 95\%) \\
\hline$<2.500 \mathrm{~g}(\mathrm{n}=43)$ & 5 & 19,6 & $10,3(-0,6-39,7)$ & 6 & 34,7 & $12,4(10,3-59,0)$ & 2 & 1,2 & $1,0(-0,8-3,2)$ & 9 & 46,2 & $11,1(24,4-67,9)$ \\
\hline $2.500 \mathrm{~g}-3.000 \mathrm{~g}(\mathrm{n}=70)$ & 4 & 0,7 & $0,4(-0,2-1,5)$ & 6 & 6,6 & $4,6(-2,5-15,7)$ & 2 & 0,4 & $0,3(-0,3-1,1)$ & 9 & 7,1 & $4,7(-2,2-16,4)$ \\
\hline$>3.000 \mathrm{~g}(\mathrm{n}=194)$ & 7 & 1,5 & $0,6(0,2-2,7)$ & 12 & 3,1 & $1,2(0,8-5,4)$ & 1 & 0,0 & $0,0(-0,1-0,1)$ & 14 & 3,2 & $1,2(0,8-5,5)$ \\
\hline Total $(n=307)$ & 16 & 2,8 & $1,2(0,5-5,1)$ & 24 & 6,7 & $2,6(1,6-11,8)$ & 5 & 0,2 & $0,2(-0,2-0,6)$ & 32 & 7,9 & $2,6(2,8-13,1)$ \\
\hline
\end{tabular}

EP: Erro padrão estimado.

Negrito: proporção estimada de déficits (todos) para as crianças com peso ao nascer inferior

a $2.500 \mathrm{~g}$ é maior (com significância estatística) do que a encontrada para as demais faixas

de peso; intervalos de confiança não se sobrepõem.

peso (menor que $2.500 \mathrm{~g}$ ) ocorreu maior prevalência de déficits nos três índices avaliados (sem significância estatística). Ao agrupar todas as formas de déficits (P/I, E/I ou P/E), verificamos que $46,2 \%$ (IC $=24,4-67,9$ ) dessas crianças tinham apresentado baixo peso ao nascer, freqüência maior e estatisticamente significante, quando comparada com as demais faixas de peso.

\section{Discussão}

\section{Limitações do estudo}

Algumas considerações devem ser feitas para uma interpretação mais adequada dos resultados deste trabalho: (a) heterogeneidade interna muito acentuada no estrato 1 devido à presença de caseiros com baixos rendimentos residindo em condomínios de alto padrão; (b) a construção de prédios de bom padrão no estrato 3, entre o ano de 1991 (quando foi realizado o censo cujos dados foram utilizados para a estratificação) e o ano de 1996 (quando se realizou a coleta de dados deste estudo), resultando em um segmento populacional com maior acesso a planos privados e manutenção de vínculos com os serviços de saúde anteriormente utilizados; (c) baixa prevalência de déficits nutricionais dificultando a análise estatística, pois as freqüências resultantes apresentaram intervalos de confiança muito amplos. As diferenças observadas talvez pudessem ser comprovadas com uma amostra maior.

\section{Os déficits nutricionais}

Neste estudo, em todos os índices antropométricos avaliados, para todas as idades, predominou o déficit estatural; o déficit ponderal foi pouco freqüente, o que corrobora a tendência 
observada em nosso país e em populações latino-americanas de baixo nível sócio-econômico (Engstrom \& Anjos, 1999; Gallo et al., 2000; Post et al., 2000). A maior prevalência de déficit ponderal observada nas crianças com baixo peso ao nascer, reforça a importância deste fator sobre o estado de saúde e as condições de sobrevivência da população infantil (Monteiro et al., 2000b; Post et al., 1996).

O declínio na prevalência de déficits nutricionais no Brasil foi bem demonstrado em inquéritos nacionais realizados em 1974 (Estudo Nacional de Despesa Familiar/ENDEF - IBGE, 1974), 1989 (Pesquisa Nacional sobre Saúde e Nutrição/PNSN - INAN, 1989) e 1996 (Pesquisa Nacional sobre Demografia e Saúde/PNDS BEMFAM, 1996). A análise desta tendência favorável, assim como de outros indicadores de saúde (Gallo, 2000), é bastante complexa. Entretanto, está claro que o desenvolvimento econômico do país não poderia explicar essa evolução, visto que as últimas duas décadas foram marcadas por períodos de estagnação econômica, desemprego e deterioração da renda. As ações e programas direcionados nas áreas da saúde e da educação têm sido apontados como importantes fatores para a evolução do perfil epidemiológico da população e melhora desses indicadores (Monteiro et al., 2000a; Monteiro \& Conde, 2000).

A ausência de estudos de base populacional anteriores a este, no Município de Embu, não permitiu que se constituísse uma série histórica do estado nutricional de sua população de crianças, mas foi possível comparar com dados de trabalhos de outras localidades e do país. Observaram-se freqüências de déficits nos índices $\mathrm{P} / \mathrm{I}(2,9 \%$; IC = 0,7-5,1) e P/E $(0,2 \%$; IC = - 0,2 $-0,6)$ inferiores às encontradas no inquérito na- cional (PNDS - BEMFAM, 1996), para o Brasil $(\mathrm{P} / \mathrm{I}=5,7 \%$ e $\mathrm{P} / \mathrm{E}=2,3 \%)$ e para o Estado de São Paulo ( $\mathrm{P} / \mathrm{I}=4,7 \%$ e $\mathrm{P} / \mathrm{E}=1,4 \%)$. Já o déficit no índice $\mathrm{E} / \mathrm{I}(7,1 \%$; IC = 1,9-12,3) foi inferior ao do Brasil (10,5\%), porém ficou próximo ao encontrado no Estado de São Paulo (6,3\%), no mesmo estudo. Embora o Município de Embu apresentasse piores indicadores sócio-econômicos e ambientais em relação ao Estado e à Região Metropolitana de São Paulo (Fundação Seade, 2001b), estes não se refletiram, nas mesmas proporções, nas condições nutricionais observadas na população infantil. Já a comparação com os dados da população de crianças menores de cinco anos, residentes na cidade de São Paulo (Monteiro \& Conde, 2000), evidenciou essa desvantagem, pois em São Paulo, no mesmo ano, a freqüência de déficits para o índice $\mathrm{E} / \mathrm{I}$ foi de $2,4 \%$.

Vários fatores poderiam estar atenuando as desvantagens da população de Embu: acesso aos serviços (Silva et al., 1997), programas de suplementação alimentar, atuação da Pastoral da Criança com ações de vigilância nutricional dirigidas a segmentos populacionais de maior risco social, entre outros. Os resultados deste estudo trouxeram importantes subsídios aos gestores locais, apontando para a necessidade de implementação de ações, em articulação com outros setores e secretarias do município, visando áreas de potencial risco, neste estudo representadas sobretudo pelo estrato 4 . Demonstraram, também, que o baixo peso ao nascer deve se manter como critério de risco para intervenções e programas de saúde (Puccini et al., 1997). Ressalta-se, ainda, que o déficit estatural deve servir como alerta e indicador de que as perdas observadas ao longo dos anos têm reflexos no futuro.

\section{Referências}

BEMFAM (Sociedade Civil Bem-Estar Familiar no Brasil), 1996. PNDS: Pesquisa Nacional sobre Demografia e Saúde. Rio de Janeiro: BEMFAM.

BUVINICH, M. R., 1998. A infância brasileira nos anos 90. In: Panorama Nutricional (C. A. Monteiro, org.), pp. 83-96, Brasília: Fundo das Nações Unidas para a Infância.

ENGSTROM, E. M. \& ANJOS, L. A., 1999. Déficit estatural nas crianças brasileiras: Relação com condições socioambientais e estado nutricional materno. Cadernos de Saúde Pública, 15:559-567. 
FREI, F., 1997. Análise de Agrupamentos: Estudo Metodológico e Aplicações em Epidemiologia, São Paulo. Dissertação de Mestrado, São Paulo: Faculdade de Saúde Pública, Universidade de São Paulo.

FUNDAÇÃO SEADE (Fundação Sistema Estadual de Análise de Dados), 2001a. Informações dos Municípios Paulistas. 25 Fevereiro 2001 <http:/ / www. seade.gov.br>.

FUNDAÇÃO SEADE (Fundação Sistema Estadual de Análise de Dados), 2001b. Pesquisa de Condições de Vida, 1998. 4 Março 2001 <http:/ / www.seade. gov.br>.

GALLO, P. R.; AMIGO, H. \& LEONE, C., 2000. Fatores de risco ao retardo de crescimento estatural em crianças de baixo nível econômico e social de São Paulo, Brasil. Archivos Latinoamericanos de $\mathrm{Nu}$ trición, 50:121-125.

IBGE (Fundação Instituto Brasileiro de Geografia e Estatística), 1974. Estudo Nacional da Despesa Familiar - ENDEF: Manual de Instruções. Rio de Janeiro: IBGE.

IBGE (Fundação Instituto Brasileiro de Geografia e Estatística), 1991. Censo Demográfico 1991. Rio de Janeiro: IBGE.

INAN (Instituto Nacional de Alimentação e Nutrição), 1989. PNSN: Pesquisa Nacional sobre Saúde e Nutrição. Perfil de Crescimento da População Brasileira de 0 a 25 anos, 1989. Brasília: INAN.

KISH, L., 1965. Survey Sampling. New York: John Wiley \& Sons.

MONTEIRO, C. A. \& CONDE, W. L., 2000. Tendência secular da desnutrição e obesidade na infância na cidade de São Paulo. Revista de Saúde Pública, 34:52-61.

MONTEIRO, C. A.; BENÍCIO, M. H. \& FREITAS, I. C. M., 2000a. Evolução da mortalidade infantil e do retardo de crescimento nos anos 90: Causas e impacto sobre as desigualdades regionais. In: Velhos e Novos Males da Saúde no Brasil. A Evolução do País e suas Doenças (C. A. Monteiro, org.), pp. 393420, 2a Ed., São Paulo: Editora Hucitec/Núcleo de Pesquisa Epidemiológica em Nutrição e Saúde, Universidade de São Paulo.

MONTEIRO, C. A.; BENÍCIO, M. H. \& ORTIZ, L. P., 2000b. Tendência secular do peso ao nascer na cidade de São Paulo (1976-1998). Revista de Saúde Pública, 34:26-40.
NCHS (National Center for Health Statistics), 1977. Growth curves for children, birth-18 years. Vital Health Statistics, 11:1-74.

ONIS, M. D.; FRONGILLO, E. A. \& BLOSSNER, M., 2000. Is malnutrition declining? Analysis of changes in levels of child malnutrition since 1980. Bulletin of the World Health Organization, 78:1222-1233.

POST, C. L. A.; VICTORA, C. G. \& BARROS, A. J. D., 2000. Entendendo a baixa prevalência de déficit de peso para estatura em crianças brasileiras de baixo nível sócio-econômico: Correlação entre índices antropométricos. Cadernos de Saúde Pública, 16:73-82.

POST, C. L.; VICTORA, C. G.; BARROS, F. C.; HORTA, B. L. \& GUIMARÃES, P. R. V., 1996. Desnutrição e obesidade infantis em duas coortes de base populacional no Sul do Brasil: Tendências e diferenciais. Cadernos de Saúde Pública, 12(Sup. 1):49-57.

PUCCINI, R. F.; WECHSLER, R.; SILVA, E. M. K. \& RESEGUE, R., 1997. Fatores de risco em crianças acompanhadas em programa de atenção à saúde. Jornal de Pediatria, 73:244-251.

SILVA, N. N.; PUCCINI, R. F.; ARAÚJO, N. S.; PEDROSO, G. C.; GOMES, M. T.; FURLANI, W. J. \& SOARES, F. S., 1997. Morbidade e Utilização de Serviços de Saúde no Município de Embu: Estudo Epidemiológico na Área da Saúde Infantil. São Paulo: Universidade Federal de São Paulo/Universidade de São Paulo/Secretaria Municipal de Saúde de Embu.

UNIFESP (Universidade Federal de São Paulo), 2001. Programa de Integração Docente-Assistencial do Embu, 2001. 13 Maio 2001 <http://www.epm.br>.

WHO (World Health Organization), 1986. Use and interpretation of anthropometric indicators of nutritional status. Bulletin of the World Health Organization, 64:929-941.

WHO (World Health Organization), 1995. Physical Status: The Use and Interpretation of Anthropometry. WHO Technical Report Series 854. Geneva: WHO.

Recebido em 31 de agosto de 2001

Versão final reapresentada em 21 de maio de 2002

Aprovado em 14 de outubro de 2002 